\title{
Carriers of Recessive WNK1/HSN2 Mutations for Hereditary Sensory and Autonomic Neuropathy Type 2 (HSAN2) Are More Sensitive to Thermal Stimuli
}

\author{
Marco L. Loggia, ${ }^{1,2}$ M. Catherine Bushnell, ${ }^{1,3,4}$ Martine Tétreault, ${ }^{6}$ Isabelle Thiffault, ${ }^{6}$ Claude Bhérer, ${ }^{6}$ \\ Nazma K. Mohammed, ${ }^{1}$ Anil A. Kuchinad, ${ }^{1}$ Audrey Laferrière, ${ }^{1}$ Marie-Josée Dicaire, ${ }^{6}$ Lina Loisel,, 6 Jeffrey S. Mogil, ${ }^{1,5}$ \\ and Bernard Brais ${ }^{6}$ \\ ${ }^{1}$ Alan Edwards Centre for Research on Pain and Departments of ${ }^{2}$ Neurology and Neurosurgery, ${ }^{3}$ Anesthesia, ${ }^{4}$ Dentistry, and ${ }^{5}$ Psychology, McGill University, \\ Montreal, Quebec, Canada H3A 2B2, and 'Laboratoire de neurogénétique de la motricité, Centre d'Excellence en Neuromique de l'Université de Montréal, \\ Centre de Recherche du Centre Hospitalier de l’Université de Montréal, Montreal, Quebec, Canada H2L 4M1
}

Hereditary sensory and autonomic neuropathy type 2 (HSAN2) is a rare recessive genetic disorder characterized by severe sensory loss affecting the tactile, thermal and nociceptive modalities. Although heterozygous carriers of nonsense mutations in the HSN2 gene, called with-no-lysine(K)-1 (WNK1), do not develop the disease, historical and experimental evidence suggests that these individuals might perceive somatosensory stimuli differently from others. Using the method-of-limits, we assessed the thresholds for warmth detection, cool detection, heat pain and cold pain in 25 mutation carriers and 35 controls. In group analyses, carriers displayed significantly lower warmth $(p<0.001)$ and cool $(p<0.05)$ difference thresholds, and also tended to report cold pain at higher temperatures $(p=0.095)$, than controls. Similarly, matched-pair analyses showed that carriers are significantly more sensitive to warm stimuli $(p<0.01)$ and cold pain stimuli $(p<0.05)$, and tend to be more sensitive to cool stimuli $(p=0.11)$. Furthermore, the differences between the warmth detection thresholds of the carriers and those of gender- and sex-matched wild types significantly increased with age $(r=0.76, p=0.02)$, and in carriers cool detection thresholds did not increase with age $(r=0.27, p=0.24)$ as expected and observed in controls $(r=0.34, p=$ 0.05). This study demonstrates that the carriers of a recessive mutation for HSAN2 display greater sensitivity to innocuous thermal stimuli, as well as for cold pain, suggesting a possible environmental adaptive advantage of the heterozygous state.

Key words: genetics; phenotype; human; psychophysics; heat; cold; pain

\section{Introduction}

Hereditary sensory and autonomic neuropathies (HSANs) are a group of clinically and genetically heterogeneous disorders associated with severe somatosensory dysfunction (Dyck and Ohta, 1975; Axelrod and Hilz, 2003; Auer-Grumbach et al., 2006). Differential diagnosis between the five main HSAN subtypes is based on their mode of inheritance, pathology, natural history, biochemical, neurophysiologic and autonomic abnormalities (Hilz, 2002). HSAN2 is an early onset (possibly congenital) recessive disorder associated with diffuse tactile deficits and a predominantly distal loss of sensitivity to thermal and painful stimuli,

Received Sept. 26, 2008; revised Dec. 17, 2008; accepted Dec. 30, 2008.

The project was funded by the Réseau de Medicine Génétique Appliquée of the Fonds de Recherche en Santé du Québec and supported by the Association de la Neuropathie Sensorielle et Autonomique Héréditaire de Type 2. M.L.L. is a Canadian Institutes of Health Research Strategic Fellow in Pain: Molecules to Community. We thank Debbie Mendelsson, Khara Sauro, Erik Jaeger, and Patrick Wood for helping with the collection of preliminary data, Maryse Robillard and Catherine Boyer for their precious help in organizing the evaluations and DNA collections, and Chris Moore for helpful discussion.

The authors declare no competing financial interests.

Correspondence should be addressed to Dr. Marco L. Loggia at his present address: Athinoula A. Martinos Center for Biomedical Imaging, Building 149, 13th Street, Room 2663, Charlestown, MA 02129-2000. E-mail: marco@nmr.mgh.harvard.edu.

DOI:10.1523/JNEUROSCI.4633-08.2009

Copyright $\odot 2009$ Society for Neuroscience $\quad$ 0270-6474/09/292162-05\$15.00/0 which lead to recurrent lacerations, fractures, and infections (Ohta et al., 1973; Nagasako et al., 2003; Roddier et al., 2005; Axelrod and Gold-von Simson, 2007). Sural nerve biopsies in HSAN2 patients reveal a severe loss of large and small myelinated fibers, and a slight reduction of unmyelinated fibers (Winkelmann et al., 1962; Ohta et al., 1973; Hilz, 2002). Compound action potentials from the sural nerve in HSAN2 patients show absent A- $\beta$ and A- $\delta$ potentials, and a diminished $\mathrm{C}$ potential (Ohta et al., 1973).

Recently, recessive nonsense mutations in the nervous system-specific HSN2 exon of WNK1 were discovered to cause the disease (Lafreniere et al., 2004; Rivière et al., 2004; Roddier et al., 2005; Cho et al., 2006; Coen et al., 2006; Takagi et al., 2006; Shekarabi et al., 2008). WNK1/HSN2 appears to be expressed in both peripheral and CNS, including satellite cells, Schwann cells, and sensory neurons (Shekarabi et al., 2008). However, the mechanisms by which these mutations cause the disease are still unclear.

The largest cluster of HSAN2 cases was uncovered in the Lanaudière region of Quebec, Canada (Roddier et al., 2005). Its occurrence corresponds to the high carrier rate of two distinct mutations in the WNK1/HSN2 gene: a common nonsense mutation, c.943C $\rightarrow$ T, and a rarer c.918-919insA insertion causing a 
frameshift (Roddier et al., 2005). Although heterozygous carriers of WNK1/HSN2 mutations do not develop the disease, the parents of many of these affected individuals spontaneously report that they seem to perceive warmth and cold differently from others. The only known quantitative sensory testing of presumed heterozygote HSAN2 French-Canadian carriers was reported in 1973 on the parents of four HSAN2 siblings: the mother showed normal touch-pressure, thermal and pain thresholds, and the father displayed normal thresholds at the level of the foot, but abnormally high tactile thresholds on the finger (Ohta et al., 1973). The large size of the extended families partaking in our study, and their regional clustering mostly to one small village in the Lanaudière region of Quebec, placed us in ideal position to explore the sensory perception of heterozygote carriers of WNK1/HSN2 mutations.

\section{Materials and Methods}

We recruited on a single day a group of age- and sex-matched carriers and noncarriers of WNK1/HSN2 mutations. Participants were asked to come to a local assembly hall where they signed a consent form approved by the Centre Hospitalier de l'Université de Montréal Institutional Ethics Committee. They were selected based on their typing for the two HSN2 mutations, which was completed during previous family and population studies. Individual genotypes were determined using Taqman SNP genotyping assay and were confirmed by direct sequencing as previously described (Roddier et al., 2005). At the time of testing, neither the participants (except for the parents of affected cases) nor the experimenters were informed of the carrier status to ensure a double-blind design. A total of 25 carriers ( 8 carrying the c. $943 \mathrm{C} \rightarrow \mathrm{T}$ and 17 carrying the c.918919insA mutation; 12 males, 13 females) and 35 wild types ( 18 males, 17 females) aged between 27 and 67 years were studied (mean age \pm SD: wild types $_{\mathrm{M}}=46.7 \pm 12$; wild types $_{\mathrm{F}}=49.6 \pm 11.6$; carriers $_{\mathrm{M}}=49.6 \pm$ 14 ; carriers $_{\mathrm{F}}=46.9 \pm 12.3$ ). Subjects in both groups were white FrenchCanadians who lived in or around the Lanaudière region of Quebec; all (including wild-type subjects) were related by blood or marriage to HSAN2 patients, or married to relatives of HSAN2 patients. All subjects were right-handed, except for one carrier and two wild types. Two female c. $943 \mathrm{C} \rightarrow \mathrm{T}$ carriers were excluded because one had suffered a stroke and another had undergone multiple upper body surgeries (all the other participants appeared in general good health, and did not have chronic pain or evidence of a peripheral neuropathy). We also excluded from the analysis of each individual test participants who were considered outliers according to the commonly adopted $1.5 \times$ interquartile (IQR) range criterion (i.e., a data point was considered an outlier if it fell either below the first quartile minus $1.5 \times \mathrm{IQR}$, or above the third quartile plus $1.5 \times$ IQR, being IQR the distance between the first and the third quartiles) (Moore and McCabe, 2003); this led to the exclusions of one carrier and one wild type from the warmth detection test and two carriers and one wild type from the cool detection test (see below). Additionally, the single carrier who was identified as an outlier in more than one test (i.e., in both warmth and cool detection tests) was excluded from the analysis of all tests, considering that he likely misunderstood the instructions. The removal of these few outliers did not significantly unbalance the age composition across groups for any test (Fig. 1 legend).

Quantitative sensory testing was performed after a 10-min period of instruction and practice with the actual experimental stimuli. The following modalities were tested in the following order, using the methodof-limits: warmth detection (WD), heat pain (HP), cool detection (CD), and cold pain $(\mathrm{CP})$ thresholds. For each test, a $30 \times 30 \mathrm{~mm}$ Peltier contact thermode (TSA II Neuro-Sensory analyzer, Medoc Ltd. Advanced Medical System), initially set to a baseline temperature of $32^{\circ} \mathrm{C}$, was applied to the skin of the volar forearm just above the wrist. When prompted by the experimenter, subjects had to press a button and hold it down until, depending on the test, they detected an increase (WD test) or a decrease (CD test) in the temperature of the thermode, or when the thermode became painfully hot (HP test) or painfully cold (CP test). The temperature change in all tests occurred randomly, after a delay of $6-8 \mathrm{~s}$ from the initial button press. The temperature change rates were $0.3^{\circ} \mathrm{C} / \mathrm{s}$ for the $\mathrm{WD}$ and $\mathrm{CD}$ tests, and $0.5^{\circ} \mathrm{C} / \mathrm{s}$ for the $\mathrm{HP}$ and $\mathrm{CP}$ tests; slow rates were selected to reduce reaction time artifacts. Each test was repeated five times, and the responses in all five trials were averaged to calculate the mean threshold for each subject. The difference threshold ( $\Delta T$; that is, the smallest change in a stimulus that can be noticed) was calculated as the absolute value of the difference of the adaptation temperature $\left(32^{\circ} \mathrm{C}\right)$ from each mean threshold value. To test as many subjects as possible, two testing stations (plus one for the practice session), each located in a separate room, were used: WD and HP were tested in the first testing station, whereas CD and CP were tested in the second. Because all the subjects were tested by the same experimenter and on the same machine for each modality, interexperimenter effects did not affect the results. To avoid sensitization or habituation to thermal stimuli after exposure to pain stimuli, sensitivity to innocuous warm/cool stimuli was always tested before sensitivity to painfully hot/cold stimuli on each stimulation site, and WD and $\mathrm{HP}$ were tested on the left forearm whereas $\mathrm{CD}$ and $\mathrm{CP}$ were tested on the right forearm.

Two types of statistical analyses were performed: a group analysis and a matched-pair analysis. In the group analysis, the performances of the two groups in each of the four tests were compared using the general linear model (GLM), including the between-subject factors genotype (carriers vs wild types) and sex, and the within-subject factor trial (1-5). Because neither a significant main effect of sex nor a significant sex $x$ trial interaction was observed, these are not considered further. The relationship between age and thresholds in each test was assessed for both genotypes using Pearson's product-moment correlation, and possible genotype differences in the correlation coefficients were statistically assessed. In the matched-pair analysis, the two genotype groups were partitioned in smaller subgroups based on the subjects' age and gender (see Table 1). The thresholds of all the subjects within the same subgroup were averaged for each test, and then the averaged thresholds of each carrier subgroup were compared with those of the age- and sex-matched wild-type subgroup. This comparison was performed using a paired $t$ test for the WD, CP, and HP tests, because the mean thresholds for these test were normally distributed (as assessed by the Shapiro-Wilks test); because the cool detection means were not normally distributed, the nonparametric Wilcoxon test was used for the CD test. The optimal number of partitions of the age range for each sex was determined to be 7 ( 1 age bin $=5.7$ years), because it was the largest number of partitions that allowed us to match each carrier subgroup with a "nonempty" wild-type subgroup (with smaller and more numerous partitions some carriers would have been included in subgroups that did not have a sex- and age-matched wild-type subgroup with an $N$ of at least 1 ). Spearman rank order correlation was used to assess the relationship between age (i.e., subgroup age range) and the differences between the averaged thresholds of matching subgroups across genotypes.

All statistical analyses were performed using Statistica 7.1 (StatSoft Inc.). Throughout the text and in the figures, means are presented \pm their SD.

\section{Results}

\section{Warmth detection test}

In the WD test, the carriers' average threshold was $33.5 \pm 0.6^{\circ} \mathrm{C}$ $\left(\Delta T=1.5^{\circ} \mathrm{C}\right)$, whereas the mean threshold in the wild types was $34.8 \pm 1.6^{\circ} \mathrm{C}\left(\Delta T=2.8^{\circ} \mathrm{C}\right)$, indicating that on average the carriers were able to detect temperature increases $1.3^{\circ} \mathrm{C}(46.4 \%)$ smaller than those detected by the wild types (Fig. 1A). Both the group analysis, $F_{(1,52)}=12.2, p<0.001$, as well as the matchedpair analysis, $t_{(8)}=3.5, p<0.01$, showed that the effect of genotype on WD thresholds is statistically significant. The correlation between WD thresholds and age (Fig. $2 A$, right) was positive but not significant in the wild-type group $(r=0.16, p=0.37)$. However, in the carriers group (Fig. $2 A$, left) the correlation between age and WD thresholds trended toward an effect in the opposite direction $(r=-0.39, p=0.08)$. The comparison between the coefficients of the WD threshold-age correlations in the two 
A

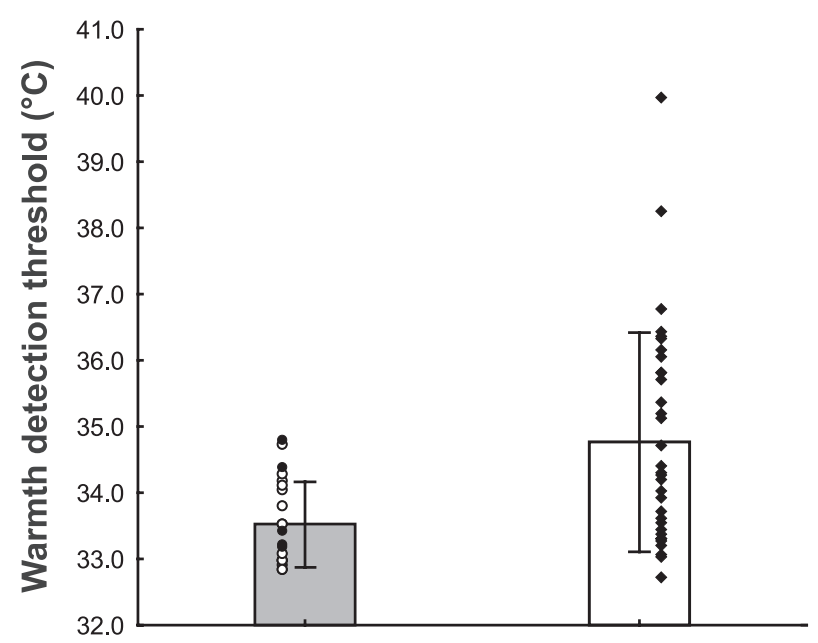

B

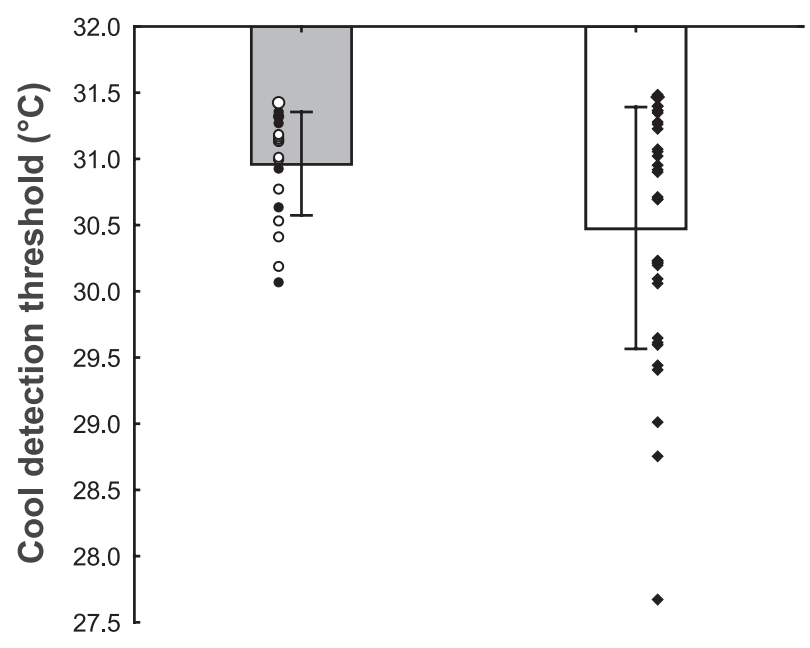

C

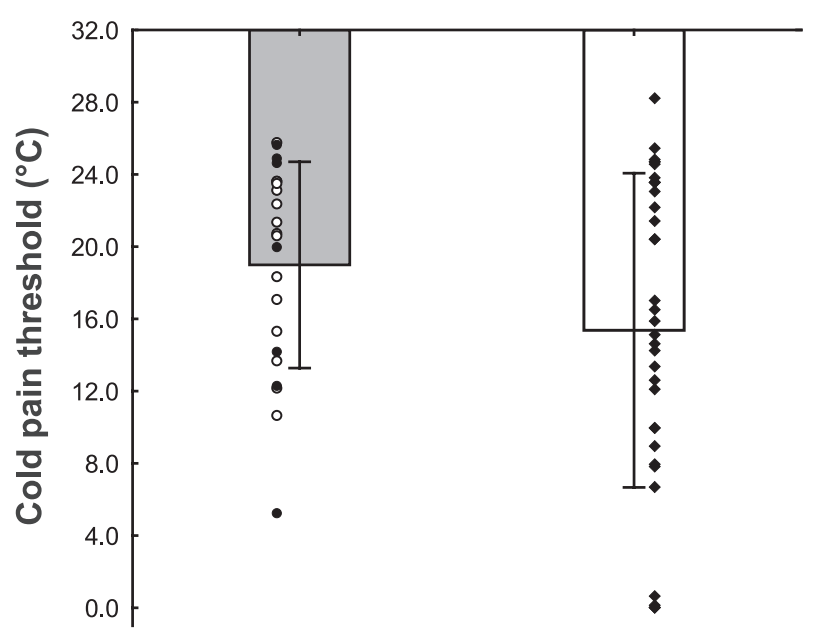

Carriers

Wildtypes
Table 1. Partitioning of the genotype groups into subgroups for the matched-pair analysis

\begin{tabular}{llll}
\hline Age range & Sex & Carriers: mean age $(M)$ & Wild type: mean age $(N)$ \\
\hline $27-32.7$ & M & $28.5(2)$ & $29(4)$ \\
& F & & \\
$32.7-38.4$ & M & & $38(1)$ \\
& F & $34.8(5)^{a}$ & $34.3(4)$ \\
$38.4-44.1$ & M & $41.7(3)$ & $39(1)$ \\
& F & & $41(3)$ \\
$44.1-49.9$ & M & $46.5(2)$ & $46.5(4)^{b}$ \\
& F & $45.5(2)$ & $48(1)$ \\
$49.9-55.6$ & M & & $54(2)$ \\
& F & & $54(1)$ \\
$55.6-61.3$ & M & $61(1)$ & $58.2(5)$ \\
& F & $58.5(2)$ & $58(5)^{c}$ \\
$61.3-67$ & M & $65.3(3)$ & $62(1)$ \\
& F & $64(2)$ & $63.7(3)$ \\
\hline
\end{tabular}

Age is expressed in years. Each group was divided into seven subgroups of male subjects and seven subgroups of female subjects. This partition allowed us to match each carrier subgroup with a nonempty wild-type subgroup of the same gender and age. M, Male; $F$, female. After the removal of the outliers: ${ }^{a} 35(4)$ in the CD test; ${ }^{b} 46.7$ (3) in the WD test; ${ }^{\prime} 58.2$ (4) in the $C D$ test.

groups yielded a $p$ value of 0.057 . Furthermore, the differences between the WD thresholds of the carriers and those of genderand sex-matched wild types significantly increased with age (Spearman $r=0.76, p=0.02$ ) (Fig. 2C). Therefore, the relationship between age and WD threshold appeared to be different between groups.

\section{Cool detection test}

The carriers displayed lower difference thresholds in the CD test as well (Fig. $1 B$ ). The mean difference threshold of the carriers was $0.5^{\circ} \mathrm{C}$ (i.e., $\left.33 \%\right)$ smaller than that of the wild types $\left(T_{\text {carriers }}\right.$ $=31.0 \pm 0.4^{\circ} \mathrm{C}$ vs $T_{\text {wild types }}=30.5 \pm 0.9^{\circ} \mathrm{C}, F_{(1,51)}=5.0, p=$ $0.03 ; \Delta T_{\text {carriers }}=1.0^{\circ} \mathrm{C}$ vs $\left.\Delta T_{\text {wild types }}=1.5^{\circ} \mathrm{C}\right)$. The matched-pair yielded similar results, although the $p$ value did not achieve the level of statistical significance $(p=0.11)$. CD difference thresholds increased with age in the wild types $(r=0.34, p=0.05)$, but not in the carriers $(r=0.27, p=0.24)$ (Fig. $2 B)$. However, neither the comparison between these correlation coefficients $(p=$ 0.82 ), nor the correlation between age and the differences between the thresholds of the carriers and matched wild types (Spearman $r=0.37, p=0.32$ ) yielded significant results.

\section{Cold pain test}

Figure $1 C$ shows that the carriers also reported $\mathrm{CP}$ at higher temperatures than the wild types $\left(T_{\text {carriers }}=19.0 \pm 5.7^{\circ} \mathrm{C}\right.$ vs $T_{\text {wild types }}$ $=15.4 \pm 8.7^{\circ} \mathrm{C}$ ). These differences trended toward significance in the group analysis, $F_{(1,53)}=2.9, p=0.10$, and reached the level of statistical significance in the matched-pair analysis, $t_{(8)}=2.75$, $p=0.03$. In the group analysis, the correlations between age and $\mathrm{CP}$ threshold were neither significant $\left(r_{\text {carriers }}=-0.09, r_{\text {wild types }}\right.$ $=0.16, p \geq 0.35)$, nor statistically different from each other ( $p=$ $0.38)$. In the matched-pair analysis the differences between carriers and matched wild types did show an increase with age

\section{$\leftarrow$}

Figure 1. Group differences. $A$, Warmth detection thresholds were significantly lower in the carriers than in the controls $(p<0.001) ; N_{\text {carriers }}=22$ (11 females), $N_{\text {wild types }}=34$ (17 females). $\boldsymbol{B}$, Carriers displayed lower cool detection threshold than the wild types $(p<0.03)$; $N_{\text {carriers }}=21$ (10 female), $N_{\text {wild types }}=34$ ( 16 females). C, Carriers tended to display lower cold detection thresholds than the wild types ( $p=0.095) ; N_{\text {carriers }}=22$ (11 females), $N_{\text {wild types }}=$ 35 (17 females). Raw data are displayed for each group. Circles, Carriers (white: c.918-919insA; black: c.934C $\rightarrow$ T); diamonds, wild types. Bars represent mean \pm SD. 
A

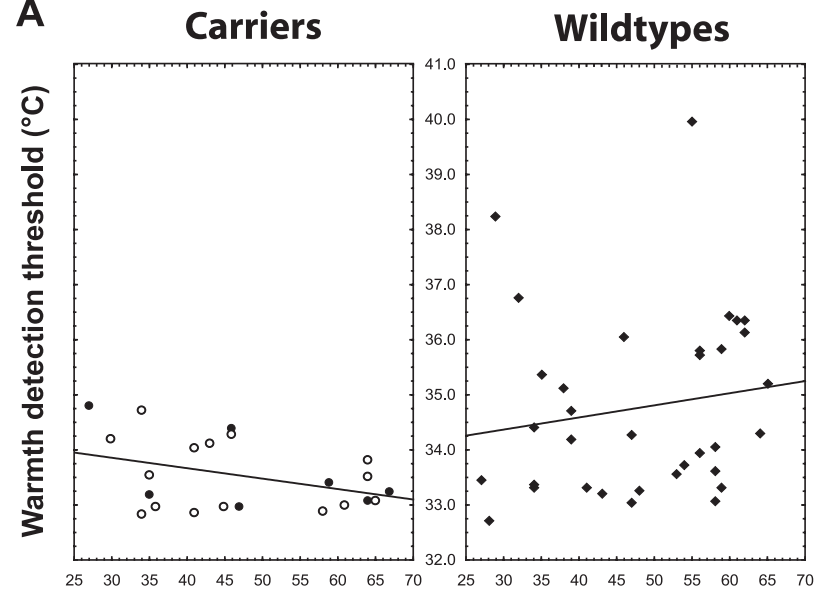

B
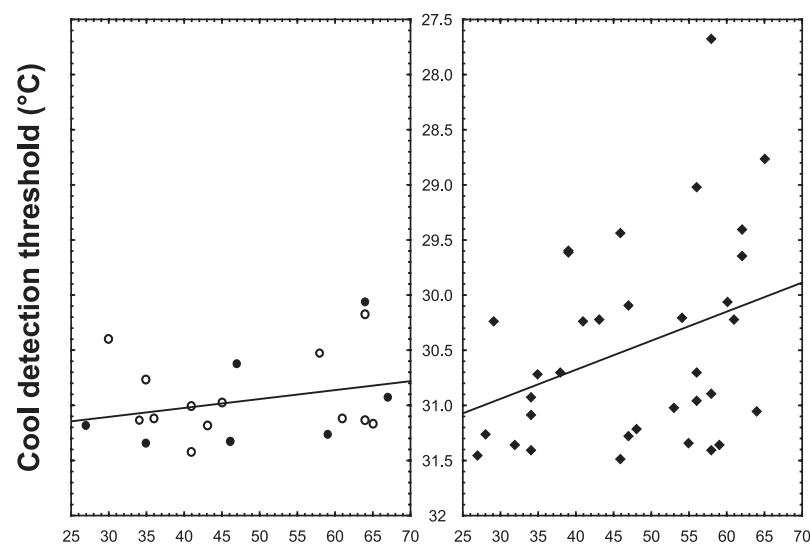

Age

C

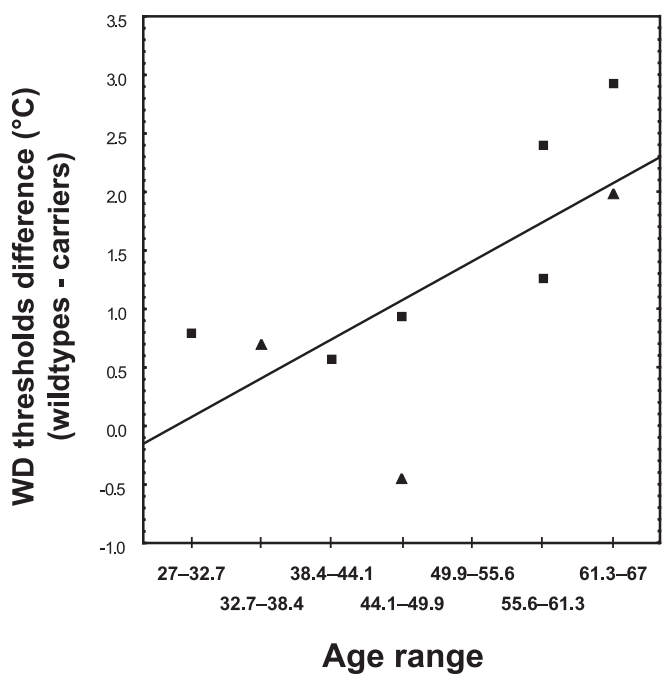

Figure 2. Correlations with age. $\boldsymbol{A}$, In the wild types (right), the correlation between WD thresholds and age was positive but not statistically significant $(r=0.16, p=0.37)$. In the carriers (left), this correlation displayed a trend in the opposite direction $(r=-0.39, p=$ 0.08). These two correlations were statistically different, $p=0.054$. $B$, Cool detection thresholds increased with age in the wild types ( $r=0.34, p=0.05$; right), but not in the carriers ( $r=$ $0.27, p=0.24$; left). However, these correlations were not statistically different ( $p=0.82$ ). See the Figure 1 legend for more information. $C$, The matched-pair analysis showed that the differences between the WD thresholds of the carriers and those of gender- and sex-matched wild types significantly increased with age (Spearman $r=0.76, p=0.02$ ). Squares, Male; triangles, female.
(Spearman $r=0.56$ ), which however did not reach statistical significance $(p=0.12)$.

\section{Heat pain test}

In the HP test, no differences were observed across genotypes, either in the group analysis $\left(T_{\text {carriers }}=41.7 \pm 3.2^{\circ} \mathrm{C}, T_{\text {wild types }}=\right.$ $\left.42.3 \pm 3.4^{\circ} \mathrm{C} ; F_{(1,53)}=0.5, p=0.50\right)$, or in the matched-pair analysis $\left(t_{(8)}=0.8, p=0.44\right)$, The correlations between age and HP threshold were neither significant $\left(r_{\text {carriers }}=-0.21, r_{\text {wild }}\right.$ types $=-0.23, p \geq 0.17$ ), nor statistically different from each other ( $p=0.91$ ). Accordingly, the differences between matching subgroups did not correlate with age (Spearman $r=-0.26, p=$ $0.50)$.

\section{Discussion}

To our knowledge this is the first study to show that carrying a recessive mutation for a peripheral sensory neuropathy may confer a potentially advantageous phenotype. Although individuals with homozygous mutations of the WNK1/HSN2 gene develop a neuropathy associated with loss of sensitivity to tactile, thermal and painful stimuli, we observed that healthy heterozygote carriers of WNK1/HSN2 mutations display lower difference thresholds for (i.e., increased sensitivity to) cool and warmth detection, as well as for cold pain, compared with wild types. Although we observed a significant overlap between the two groups, the wild types' thresholds were distributed over a larger range. For instance, only $\sim 60 \%$ of wild types were able to detect temperature increases smaller than $3^{\circ} \mathrm{C}$, as opposed to $100 \%$ of carriers; similarly, only $\sim 75 \%$ of the wild types, compared with $100 \%$ of the carriers, detected temperatures decreases smaller than $2^{\circ} \mathrm{C}$. Furthermore, we show that carriers do not appear to display the age-related decline in somatosensory sensibility observed in wildtype individuals: although the younger subjects in both groups displayed similar thresholds, the differences between the warmth detection thresholds of the carriers and those of gender- and sex-matched wild types significantly increased with age, and cool detection thresholds did not increase with age in carriers, as expected and observed in wild types. The two types of analysis used in this study, group and matched-pair analyses, yielded very similar results but also presented some differences: in particular, the former analysis produced significant $p$ values for the $\mathrm{WD}$ and $\mathrm{CD}$ tests, whereas the $p$ value for the $\mathrm{CP}$ test approached significance; the latter analysis produced $p$ values that were significant for the WD and CP tests, but only approached significance for the CD test. Because in our study it was not possible to exactly match each carrier with one wild-type subject, we had to average a large amount of data (i.e., all the datapoints within each subgroup, for each test) to perform the matched-pair analysis. Although this procedure significantly reduced the degrees of freedom of our analysis (hence the higher $p$ values in WD and CD tests), it allowed the unveiling of an effect (i.e., in the CP test) that in the group analysis only approached significance, likely due to its high intersubject variability.

Although this is the first time that recessive mutations responsible for a neuropathy have been associated with a potentially beneficial phenotype, examples of recessive mutations leading to advantageous traits have been identified in other domains (e.g., protection against certain forms of infections; Segal and Hill, 2003). It is of interest that most cases published to date have been found in countries with cold climates, such as Canada. The high carrier frequency in French Canada suggests that settlers that carried these mutations saw their descendants prosper in the cold environment, further supporting a possible environmental ad- 
vantage to the heterozygous state. By conferring an increased thermal sensitivity, these mutations might allow the carriers to 'fine-tune' their somatosensory function to the extreme environments they live in, by counteracting the desensitizing effects that low ambient temperatures exert on the perception of both innocuous and noxious thermal stimuli (Strigo et al., 2000). However, because the testing sessions described in this study have been performed at room temperature, further studies need to be performed in cold ambient temperatures to further support this hypothesis.

The physiological mechanisms by which carrying a single copy of a predicted truncated WNK1 leads to an enhanced thermal sensitivity, whereas carrying both truncated copies leads to congenital insensitivity to thermal, pain and tactile stimuli, still remain to be investigated, and could potentially range from factors affecting membrane conductance within primary sensory neurons, up to high-order cognitive factors. For instance, one possibility is that the truncation of this protein might lead to an increase in the membrane excitability of the primary sensory neurons: in carriers of a single truncated copy of the gene, this increased excitability might be mild and result in a leftward shift in the stimulus-response curve and a lowering of the spiking threshold; in homozygous carriers, however, this increased excitability might be so dramatic to induce excitotoxicity, and therefore lead to the diminution of primary sensory neurons that has been observed in HSAN2 patients (Winkelmann et al., 1962; Ohta et al., 1973; Hilz, 2002). However, in the absence of further data, any explanation remains purely speculative. The generation of cellular and animal models and a better understanding of the role of WNK1/HSN2 in the sensory nervous system development and maintenance should shed light on the beneficial nature of the perceptual changes in carriers, as well as on the pathogenesis of HSAN2.

\section{References}

Auer-Grumbach M, Mauko B, Auer-Grumbach P, Pieber TR (2006) Molecular genetics of hereditary sensory neuropathies. Neuromolecular Med 8:147-158.

Axelrod FB, Gold-von Simson G (2007) Hereditary sensory and autonomic neuropathies: types II, III, and IV. Orphanet J Rare Dis 2:39.

Axelrod FB, Hilz MJ (2003) Inherited autonomic neuropathies. Semin Neurol 23:381-390.

Cho HJ, Kim BJ, Suh YL, An JY, Ki CS (2006) Novel mutation in the HSN2 gene in a Korean patient with hereditary sensory and autonomic neuropathy type 2. J Hum Genet 51:905-908.

Coen K, Pareyson D, Auer-Grumbach M, Buyse G, Goemans N, Claeys KG,
Verpoorten N, Laur M, Scaioli V, Salmhofer W, Pieber TR, Nelis E, De Jonghe P, Timmerman V (2006) Novel mutations in the HSN2 gene causing hereditary sensory and autonomic neuropathy type II. Neurology 66:748-751.

Dyck PJ, Ohta M (1975) Neuronal atrophy and degeneration predominantly affecting peripheral sensory neurons. In: Peripheral neuropathy, Vol 2 (Dyck PJ, Thomas PK, Lambert EH, eds), pp 791-824. Philadelphia: WB Saunders.

Hilz MJ (2002) Assessment and evaluation of hereditary sensory and autonomic neuropathies with autonomic and neurophysiological examinations. Clin Auton Res 12 [Suppl 1]:I33-I43.

Lafreniere RG, MacDonald ML, Dube MP, MacFarlane J, O’Driscoll M, Brais B, Meilleur S, Brinkman RR, Dadivas O, Pape T, Platon C, Radomski C, Risler J, Thompson J, Guerra-Escobio AM, Davar G, Breakefield XO, Pimstone SN, Green R, Pryse-Phillips W, et al (2004) Identification of a novel gene (HSN2) causing hereditary sensory and autonomic neuropathy type II through the study of Canadian genetic isolates. Am J Hum Genet 74:1064-1073.

Moore DS, McCabe GP (2003) Introduction to the practice of statistics. New York: W. H. Freeman.

Nagasako EM, Oaklander AL, Dworkin RH (2003) Congenital insensitivity to pain: an update. Pain 101:213-219.

Ohta M, Ellefson RD, Lambert EH, Dyck PJ (1973) Hereditary sensory neuropathy, type II. Clinical, electrophysiologic, histologic, and biochemical studies of a Quebec kinship. Arch Neurol 29:23-37.

Rivière JB, Verlaan DJ, Shekarabi M, Lafrenière RG, Bénard M, Der Kaloustian V, Shbaklo Z, Rouleau GA (2004) A mutation in the HSN2 gene causes sensory neuropathy type II in a Lebanese family. Ann Neurol 56:572-575.

Roddier K, Thomas T, Marleau G, Gagnon AM, Dicaire MJ, St-Denis A, Gosselin I, Sarrazin AM, Larbrisseau A, Lambert M, Vanasse M, Gaudet D, Rouleau GA, Brais B (2005) Two mutations in the HSN2 gene explain the high prevalence of HSAN2 in French Canadians. Neurology 64:1762-1767.

Segal S, Hill AV (2003) Genetic susceptibility to infectious disease. Trends Microbiol 11:445-448.

Shekarabi M, Girard N, Rivière JB, Dion P, Houle M, Toulouse A, Lafrenière RG, Vercauteren F, Hince P, Laganiere J, Rochefort D, Faivre L, Samuels M, Rouleau GA (2008) Mutations in the nervous system-specific HSN2 exon of WNK1 cause hereditary sensory neuropathy type II. J Clin Invest 118:2496-2505.

Strigo IA, Carli F, Bushnell MC (2000) Effect of ambient temperature on human pain and temperature perception. Anesthesiology 92:699-707.

Takagi M, Ozawa T, Hara K, Naruse S, Ishihara T, Shimbo J, Igarashi S, Tanaka K, Onodera O, Nishizawa M (2006) New HSN2 mutation in Japanese patient with hereditary sensory and autonomic neuropathy type 2. Neurology 66:1251-1252.

Winkelmann RK, Lambert EH, Hayles AB (1962) Congenital absence of pain. Report of a case and experimental studies. Arch Dermatol 85:325339. 